Article

\title{
On the Potential of Silicon Intermediate Band Solar Cells
}

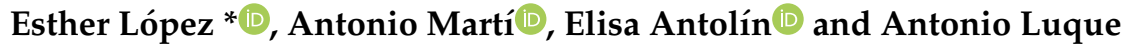 \\ Instituto de Energía Solar, Universidad Politécnica de Madrid, Avenida Complutense 30, 28040 Madrid, Spain; \\ antonio.marti@upm.es (A.M.); elisa.antolin@upm.es (E.A.); a.luque@upm.es (A.L.) \\ * Correspondence: esther.lopez@ies.upm.es
}

Received: 19 May 2020; Accepted: 10 June 2020; Published: 12 June 2020

\begin{abstract}
Intermediate band solar cells (IBSCs) have an efficiency limit of $63.2 \%$, which is significantly higher than the $40.7 \%$ limit for conventional single gap solar cells. In order to achieve the maximum efficiency, the total bandgap of the cell should be in the range of $\sim 2 \mathrm{eV}$. However, that fact does not prevent other cells based on different semiconductor bandgaps from benefiting from the presence of an intermediate band (IB) within their bandgap. Since silicon (1.12 eV bandgap) is the dominant material in solar cell technology, it is of interest to determine the limit efficiency of a silicon IBSC, because even a modest gain in efficiency could trigger a large commercial interest if the IB is implemented at low cost. In this work we study the limit efficiency of silicon-based IBSCs considering operating conditions that include the use of non-ideal photon casting between the optical transitions, different light intensities and Auger recombination. The results lead to the conclusion that a silicon IBSC, operating under the conventional model in which the sub-bandgaps add to the total silicon gap, provides an efficiency gain if operated in the medium-high concentration range. The performance of these devices is affected by Auger recombination only under extremely high concentrations.
\end{abstract}

Keywords: intermediate band solar cells; silicon; limit efficiency; non-overlapped coefficients; Auger recombination

\section{Introduction}

The idea behind the intermediate band solar cell (IBSC) [1,2] concept is the absorption of sub-bandgap energy photons to produce electric work. These photons are absorbed by engineering a semiconductor-like material that, in addition to the conduction and valence bands, exhibits an intermediate band (IB) within the otherwise conventional semiconductor gap.

The basic structure of an IBSC is illustrated in Figure 1, where the (IB) is shown splitting the total bandgap of the semiconductor, $E_{G}$, into two sub-bandgaps, $E_{L}$ (the low energy sub-bandgap) and $E_{H}$ (the high energy sub-bandgap). The absorption of sub-bandgap photons is illustrated in Figure 1 by the arrows labeled a1 and a2. This absorption produces an increase in cell photocurrent, providing a gain in efficiency if the output voltage is not limited by any of the sub-bandgaps $E_{L}$ nor $E_{H}$. The IB is isolated from the metal contacts by means of conventional semiconductors (called emitters) in order to assure that the output voltage is limited by $E_{G}$. 


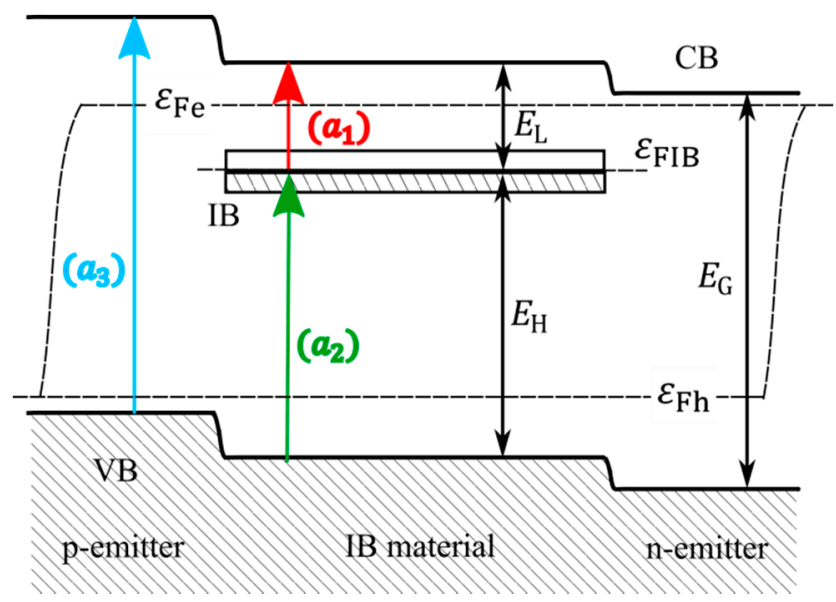

Figure 1. Energy band diagram of an intermediate band solar cell (IBSC). The three energy bands and the absorption processes related to them are shown.

Concerning the properties of the IB, we will keep our work within the conventional framework that considers a non-ratchet IB. In the non-ratchet framework, the optical gaps are constrained to $E_{L}+E_{H}=E_{G}$. The "photon-ratchet" intermediate band solar cell proposed by Yoshida et al. in 2012 [3] improves the conventional model by distinguishing between different energy states within the IB so that, for example, transitions a2 take place from the valence band (VB) to upper states in the IB (which defines the optical bandgap $E_{H}$ ) and transitions a1 take place between lower states in the IB to the conduction band (CB) (which defines the optical bandgap $E_{L}$ ) so that $E_{L}+E_{H} \neq E_{G}$.

An approach to implement IB materials consists of introducing a high concentration of impurities inside the lattice of a host semiconductor. The impurity-host interaction can result in the emergence of deep-levels (DLs) inside the semiconductor bandgap, which usually act as non-radiative recombination centers. However, if the impurity concentration is high enough to produce Mott transition [4], the wave function of the DLs can be delocalized, forming an IB [5]. There is some controversy about whether sufficiently increasing the concentration of impurities can reduce the non-radiative recombination related to DLs or not, with theoretical works that support this possibility [6,7] and theoretical works that do not [8]. To test experimentally the limitations of the DLs approach well-known semiconductors such as silicon have been studied, despite its low bandgap $(\sim 1.12 \mathrm{eV})$ when compared to the optimum one $(1.95 \mathrm{eV})$ [1] for IBSC operation. Several works suggest that an IBSC based on silicon could be implemented in practice by inserting impurities of transition metals such as titanium (Ti) [9-11], cobalt (Co) [12], selenium (Se) [13,14] and sulfur (S) [14-17]. This is supported by experimental results that prove some of the features related to the IBSC operation, such as sub-bandgap absorption [14], lifetime recovery [16-18] and splitting of quasi Fermi levels between the IB and the CB [19].

In spite of this controversy, since silicon is the dominant material in solar cell technology, it is important to clarify whether silicon IBSC can provide an efficiency gain, since even a modest increase in efficiency could trigger a large commercial interest. For this reason, the objective of this work is to determine the efficiency of silicon-based non-ratchet IBSCs. To this end, we will consider not only the ideal radiative limit case, but also a more realistic scenario for the case of silicon that includes Auger non-radiative recombination, unavoidable even in defect-free semiconductors. Non-ideal photon casting between the optical transitions will also be considered. The case in which the optical gaps are not constrained to $E_{L}+E_{H}=E_{G}$, operating in the radiative limit, can be found in the work by Krishna and J. J. Krich [20].

\section{Materials and Methods}

The model to determine the radiative limiting efficiency of an IBSC was first formulated in reference [1]. This model assumed "non-overlapping" of the absorption coefficients, which means 
that the absorption of photons with a specific energy can only take place in one electronic transition. In particular, to achieve the limit efficiency of an IBSC the absorption coefficients have to be tailored in a way that a photon with energy $E_{\mathrm{ph}}$, high enough to be absorbed by means of several inter-band transitions, is absorbed in the electronic transition involving the highest bandgap. By imposing this condition, the thermalization losses $\left(E_{\text {loss }}\right)$ resulting from absorbing photons with excessive energy (with an energy higher than the involved inter-band transition) are minimized. Therefore, when investigating the maximum efficiency of IBSCs, the condition of non-overlapping absorption coefficients is imposed and the values of all the bandgaps are optimized in order to seek this maximum. However, when the value of all the bandgaps is fixed and determined by the fabrication process, as it would be in the case of IBSCs based on silicon, an increase in the thermalization losses resulting from overlapping absorption coefficients (illustrated in Figure 2d) can be compensated by a better current matching in the IBSC [20].

(a)

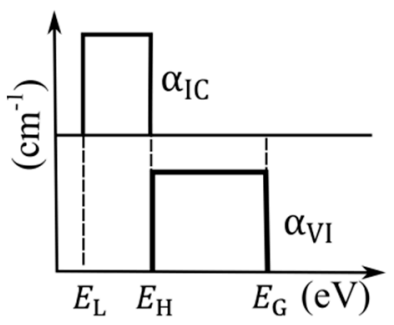

(c)

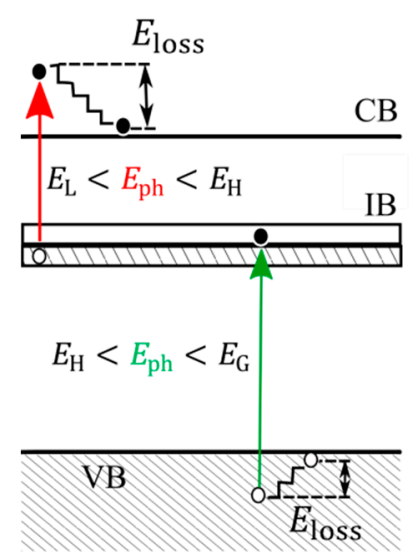

(b)

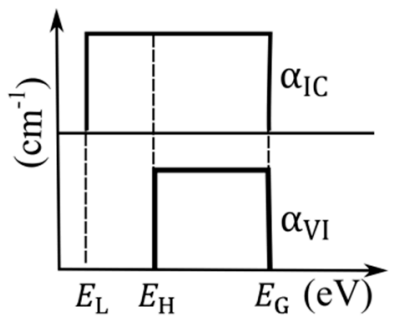

(d)

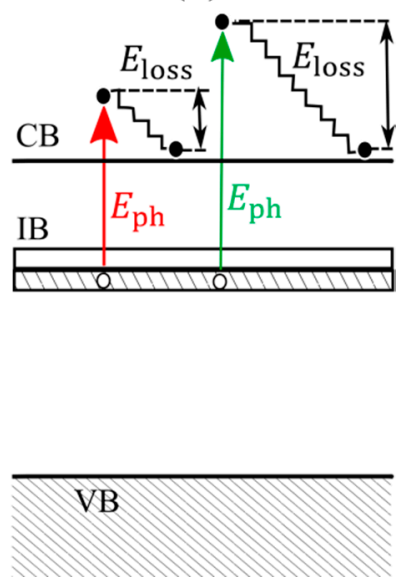

Figure 2. Illustration of non-overlapped (a) and overlapped (b) absorption coefficients corresponding to inter-band transitions from the IB to the $\mathrm{CB}\left(\alpha^{I C}\right)$ and from the VB to the IB $\left(\alpha^{V I}\right)$. The resulting photon absorption processes are illustrated $(\mathbf{c}, \mathbf{d})$, separately, together with the corresponding thermalization losses $\left(E_{\text {loss }}\right)$.

The mathematical model for IBSCs with overlapping absorption coefficients was described in reference [21]. We use this model, based on the detailed balance, to study the limiting efficiency of silicon IBSCs, assuming, for simplification purposes, the sun is a black body at $6000 \mathrm{~K}\left(T_{S}\right)$ and the cell and its surroundings are black bodies at $300 \mathrm{~K}\left(T_{C}\right)$. In this context, the general equation describing the current-voltage $(J-V)$ characteristic is given by:

$$
J(V)=e \int_{E} M_{e}\left(F_{a b s}-F_{e m}\right) d E-J_{B, e}=e \int_{E} M_{h}\left(F_{a b s}-F_{e m}\right) d E-J_{B, h}
$$


where $E$ is the photon energy, and factors $M_{e}$ and $M_{h}$, which can be considered equivalent to electron and hole absorptivity, are expressed as

$$
\begin{aligned}
& M_{e}=\frac{\alpha_{V C}+\alpha_{I C}}{\alpha_{V C}+\alpha_{I C}+\alpha_{V I}} \\
& M_{h}=\frac{\alpha_{V C}+\alpha_{V I}}{\alpha_{V C}+\alpha_{I C}+\alpha_{V I}}
\end{aligned}
$$

$\alpha_{X Y}$ is the photon absorption coefficient related to the transition from the $\mathrm{X}$ band to the $\mathrm{Y}$ band; $e$ is the electron charge; $F_{a b s}$ is the photon flux absorbed by the cell (which equals the incoming photon flux); $F_{e m}$ is the photon flux emitted by the cell; and $J_{B, e}$ and $J_{B, h}$ are loss factors. Those loss factors are associated with the irreversibility due to photons emitted as a consequence of a radiative transition from band $Y$ to band $X$ being reabsorbed, not by means of an absorption from band $X$ to band $Y$ but as a transition in another band.

As explained in [21], when the absorption coefficients overlap, the loss factors $J_{B, e}$ and $J_{B, h}$ depend also on the cell thickness and can be considered arbitrarily small when the cell thickness tends to zero. In the case of silicon, the use of light trapping techniques [22,23] makes it plausible to consider $J_{B, e}=J_{B, h}=0$ as a limiting case to calculate the cell limiting efficiency. The absorption enhancement obtained with these techniques allows assuming that every photon with energies higher than $E_{L}$ is absorbed, so that an absorptivity equal to 1 can be supposed. To simplify the study, we shall also assume that the absorption coefficients $\alpha_{I C}$ and $\alpha_{V I}$ can overlap but they do not overlap with $\alpha_{V C}$. This hypothesis can be considered as an approximation describing the working case in which photons capable of causing transitions from the valence band to the conduction band are absorbed in the front emitter of the cell (as illustrated in Figure 1) leaving for the IB material the task of dealing with the absorption of below bandgap photons. Under this hypothesis, $M_{e}$ and $M_{h}$ are given by:

$$
\begin{gathered}
M_{e}=\left\{\begin{array}{c}
1 \text { if } E_{L}<\varepsilon<E_{H} \\
\frac{\alpha_{I C}}{\alpha_{I C}+\alpha_{V I}} \equiv \text { if } E_{H}<\varepsilon<E_{G} \\
1 \text { if } \varepsilon>E_{G}
\end{array}\right. \\
M_{h}=\left\{\begin{array}{c}
0 \text { if } E_{L}<\varepsilon<E_{H} \\
\frac{\alpha_{V I}}{\alpha_{I C}+\alpha_{V I}} \equiv \begin{array}{l}
1-\gamma \quad \text { if } E_{H}<\varepsilon<E_{G} \\
1 \text { if } \varepsilon>E_{G}
\end{array}
\end{array}\right.
\end{gathered}
$$

and, Equation (1) is expanded as

$$
\begin{aligned}
& J(V)=\pi \sin ^{2} \theta_{S} X \dot{N}\left(E_{L}, E_{H}, 0, T_{S}\right)+\pi\left(1-\sin ^{2} \theta_{S} X\right) \dot{N}\left(E_{L}, E_{H}, 0, T_{C}\right) \\
& -\pi \dot{N}\left(E_{L}, E_{H}, \mu_{C I}, T_{C}\right)+\pi \gamma \sin ^{2} \theta_{S} X \dot{N}\left(E_{H}, E_{G}, 0, T_{S}\right) \\
& +\pi \gamma\left(1-\sin ^{2} \theta_{S} X\right) \dot{N}\left(E_{H}, E_{G}, 0, T_{C}\right)-\pi \gamma \dot{N}\left(E_{H}, E_{G}, \mu_{C I}, T_{C}\right) \\
& +\pi \sin ^{2} \theta_{S} X \dot{N}\left(E_{G}, \infty, 0, T_{S}\right)+\pi\left(1-\sin ^{2} \theta_{S} X\right) \dot{N}\left(E_{G}, \infty, 0, T_{C}\right) \\
& -\pi \dot{N}\left(E_{G}, \infty, \mu_{C V}, T_{C}\right)
\end{aligned}
$$

where $X$ is the solar concentration, $\sin ^{2} \theta_{S}=1 / 46050$ corresponds to the semi-angle of the sun disc seen from Earth and

$$
\dot{N}\left(E_{1}, E_{2}, \mu, T\right)=\frac{2}{h^{3} c^{2}} \int_{E_{1}}^{E_{2}} \frac{E^{2}}{\exp \left(\frac{E-\mu}{k_{\mathrm{B}} T}\right)-1} d E
$$

describes the flux of photons per unit of etendùe at temperature $T$ and chemical potential $\mu$ for photons with energy between $E_{1}$ and $E_{2}$. In Equation (7) $c$ is the speed of light in vacuum, $h$ is the Planck constant and $k_{\mathrm{B}}$ is the Boltzmann constant. For a given voltage $(V)$ the following condition must be fulfilled:

$$
e V=\mu_{C V}=\mu_{I V}+\mu_{C I}
$$


where $\mu_{C V}, \mu_{C I}$ and $\mu_{I V}$ are the chemical potentials of photons involved in transitions between the $\mathrm{VB}$ and the $\mathrm{CB}$; the IB and the $\mathrm{CB}$; and the VB and the IB, respectively. The presence of the factor "-1" in Equation (7), which ultimately derives from the inclusion of stimulated emission in the model [24], imposes that the value of those chemical potentials, which equals the splitting of the quasi-Fermi levels, cannot be larger than the bandgap involved in the electronic transitions (that is, $\mu_{C V}<E_{G}, \mu_{I V}<E_{H}$, and $\left.\mu_{C I}<E_{L}\right)$. Assuming that the IB has a high density of states, the quasi-Fermi level of the IB can be fixed within this energy band (illustrated in Figure 1), enabling both empty states to receive electrons from the VB as filled states to supply electrons to the CB.

Equation (1) assumes that only radiative recombination takes place between bands. However, it is well known that silicon cells are affected by non-radiative recombination like Auger recombination, which is the predominant recombination mechanism when silicon cells are illuminated with concentrated light. For this reason, it is worth including this non-radiative recombination mechanism in order to know whether the efficiency potential of an IBSC based on silicon still remains under more realistic circumstances.

In order to include Auger recombination, Equation (1) must be modified as follows:

$$
J(V)=e \int_{E} M_{e}\left(F_{a b s}-F_{e m}\right) d E-J_{B, e}-J_{\mathrm{Au}}=e \int_{E} M_{h}\left(F_{a b s}-F_{e m}\right) d E-J_{B, h}-J_{\mathrm{Au}}
$$

where $J_{A u}$ is the Auger recombination current. This current is calculated assuming flat quasi-Fermi levels, which leads to the following equations:

$$
\begin{aligned}
& J_{\mathrm{Au}}=e W\left(C_{\mathrm{e}} n+C_{\mathrm{h}} p\right) n_{\mathrm{i}}{ }_{\mathrm{i}}\left(\exp \frac{e V}{k T}-1\right)=e n_{i}{ }^{2} W C_{\mathrm{e}} N_{C} \exp \frac{-E_{L}}{k T} \exp \frac{\mu_{C I}}{k T}\left(\exp \frac{e V}{k T}-1\right) \\
& +e n_{i}{ }^{2} W C_{\mathrm{h}} N_{V} \exp \frac{-E_{H}}{k T} \exp \frac{\mu_{I V}}{k T}\left(\exp \frac{e V}{k T}-1\right)
\end{aligned}
$$

In this expression, $W$ is the thickness of the cell; $n, p$ and $n_{i}$ are electron, holes and intrinsic concentration, respectively; and $C_{\mathrm{e}}$ and $C_{\mathrm{h}}$ are Auger coefficients [25].

With the conditions mentioned above, Equation (6) is numerically solved in order to find $\mu_{C I}$ and $\mu_{I V}$ and, therefore, the current voltage-characteristic $J(V)$. Once this current-voltage characteristic is solved, the maximum power point and the efficiency of IBSCs can be calculated.

\section{Results and Discussion}

The efficiency limit of silicon-based IBSCs is calculated with the model and considerations described above. To this end the $J(V)$ characteristic is numerically solved, with Equation (6) for the radiative limit and Equation (9) when including Auger recombination. Notice that, in Equation (6), the $J(V)$ characteristic depends on $\mu_{C I}$ but not on $\mu_{I V}$. This is because the total current density $(J)$ is defined in terms of the electron current density. $J$ can also be defined as a function of the hole current density with an equation similar to Equation (6), but replacing $E_{L}$ with $E_{H}, \gamma$ with $1-\gamma$ and $\mu_{C I}$ with $\mu_{I V}$. By imposing that electron and hole current density must be equal, the values for $\mu_{C I}$ and $\mu_{I V}$ can be obtained, and consequently the $J(V)$ characteristic, the maximum power point and the efficiency of IBSCs can be calculated. The results are obtained as a function of the $E_{L}$, swiping the value of this parameter from $0 \mathrm{eV}$ to half of the silicon bandgap, since symmetry with respect to the mid-gap is expected.

Results are plotted in Figures 3 and 4. In those figures the curves labeled "Non-overlapping" correspond to absorption coefficients that do not overlap $(\gamma=0)$. Curves labeled "Overlapping" correspond to absorption coefficients $\alpha_{I C}$ and $\alpha_{V I}$ that do overlap $(0<\gamma \leq 1)$, so for each $E_{L}, \gamma$ is optimized for maximum efficiency. Curves labeled as "Auger" plot the results obtained using Equation (9). For the calculus, cell thicknesses of $W=200 \mu \mathrm{m}$ and $W=50 \mu \mathrm{m}$ are assumed and we still consider $J_{B, e}=0$ and $J_{B, h}=0$ (neglecting losses related to inefficient photon recycling) in order to provide an upper bound of the limit efficiency. The study includes several solar concentrations: One sun (Figure 3), 100 suns and the maximum concentration of 46,050 suns (Figure 4). 


\subsection{One-Sun Case}

Silicon solar cells are mostly used in flat modules operating under irradiances in the one-sun range. Therefore, studying the limiting efficiency of silicon IBSCs under these circumstances is our first goal. From the results plotted in Figure 3, it can be noticed that at one sun there is no efficiency gain for a non-ratched IBSC over the single gap solar cell. The limit efficiency of a silicon non-ratched IBSC is not higher than the limit efficiency of a single gap silicon solar cell, and only when the IB is located at the middle of the gap and the absorption coefficients do not overlap does it equals the limit efficiency of a single gap silicon cell. This is because under those circumstances the IB does not contribute at all, neither to the enhancement of the photocurrent nor to the radiative recombination, so the operation of an IBSC is equivalent to the one of a single gap cell. In this work the efficiency of the silicon cell is not improved by the inclusion of an IB due to the restrictions imposed on the slitting of the quasi-Fermi level. However, it must be pointed out that imposing different conditions like absorption edges whose value adds up to more than the bandgap involved [20] or different energy band diagrams can still enable an efficiency gain [26].

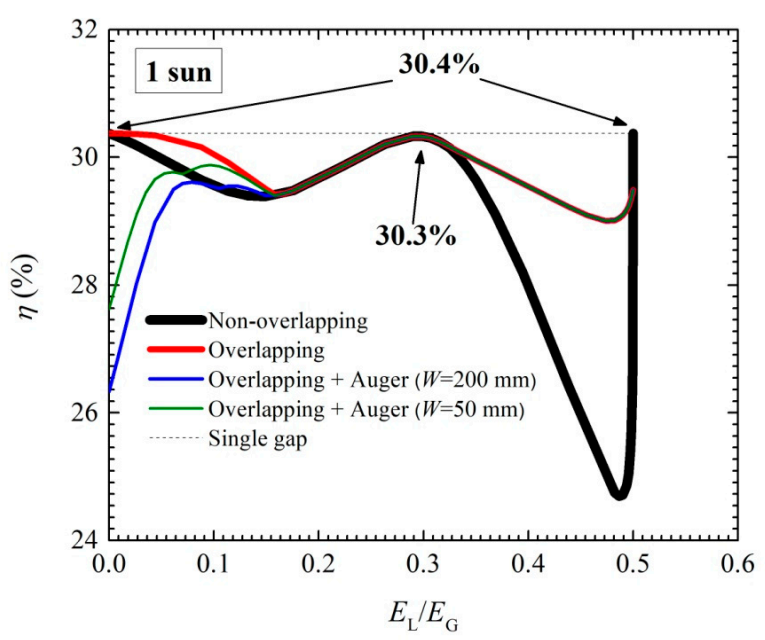

Figure 3. Limit efficiency of silicon IBSC at one sun for different IB positions with and without overlapping and with and without Auger recombination (discussed in Section 3.3). The value of $\gamma$ is optimized to maximize the efficiency at every $E_{L}$ and the limit efficiency of a conventional silicon solar cell is shown for comparison.

Results from Figure 3 corroborate what we advanced previously regarding that the efficiency limit of IBSCs with non-optimum bandgaps might be higher with overlapped absorption coefficients. In particular, this is true when $0<E_{L} / E_{G} \leq 0.16$ (for $\gamma=0.99$ ) and $0.33 \leq E_{L} / E_{G}<0.5$ (for $0.11 \leq \gamma \leq 0.5$ ). The reason is an enhancement in the current generation due to a better match between the carriers generated in inter-band transitions from the IB to the $\mathrm{CB}$ and from the VB to the IB, as a consequence of the overlapping of the corresponding absorption coefficients $\left(\alpha_{I C}\right.$ and $\left.\alpha_{V I}\right)$.

\subsection{Concentration Case}

The situation changes when concentration is used. At 100 suns the limiting efficiency of silicon IBSC surpasses the limiting efficiency of a single gap silicon solar cell (Figure 4 top). At this concentration, the maximum limiting efficiency of the IBSC reaches $41.8 \%$ when the IB is located at an optimum position $\left(E_{L} / E_{G}=0.31\right)$, being significantly higher than the $34.82 \%$ of a silicon cell without IB. Overlapping absorption coefficients provide a higher limiting efficiency that the non-overlapping ones when $E_{L} / E_{G}$ $>0.31$ and $0 \leq \gamma \leq 0.5$. However, those efficiencies do not surpass the maximum efficiency obtained with non-overlapping absorption coefficients and an optimum IB. This is different to what is reported in reference [20], where the highest efficiency is obtained with overlapping absorption coefficients 
(also at one sun) but for an IB with optical gaps not constrained to $E_{L}+E_{H}=E_{G}$, as anticipated in the introduction.

At maximum concentration (46050 suns), the obtained results show a similar behavior as in the case of 100 suns. The silicon IBSC increases its limiting efficiency with respect to the cell without IB, but in this case for every value of $E_{L} / E_{G}$ (Figure 4 bottom). Silicon IBSC obtains a maximum efficiency of $54.6 \%$ compared with $40.7 \%$ for bare silicon. The highest efficiency is obtained when $E_{L} / E_{G}=0.32$. As in the previous case, for $E_{L} / E_{G}$ above 0.32 , overlapping absorption coefficients provide higher efficiency (for $0 \leq \gamma \leq 0.5$ ) than the non-overlapping ones.

An important conclusion worth emphasizing from these results is that IBSCs with overlapping absorption coefficients exhibit higher limiting efficiency when $E_{L}$ is higher than the optimum value. This result draws attention to the fact that, when dealing with non-optimum bandgaps, an IBSC in which overlap exists can give more efficiency than the case in which overlap does not exist. Therefore, the fact that the condition of non-overlapping is required for attaining the IBSC with the absolute maximum efficiency must not mislead researchers to pursue the same condition when non-ideal bandgaps are used. In addition, these results imply that pursuing silicon IBSCs with optical gaps constrained to $E_{L}+E_{H}=E_{G}$ only makes sense if used under concentrated light.
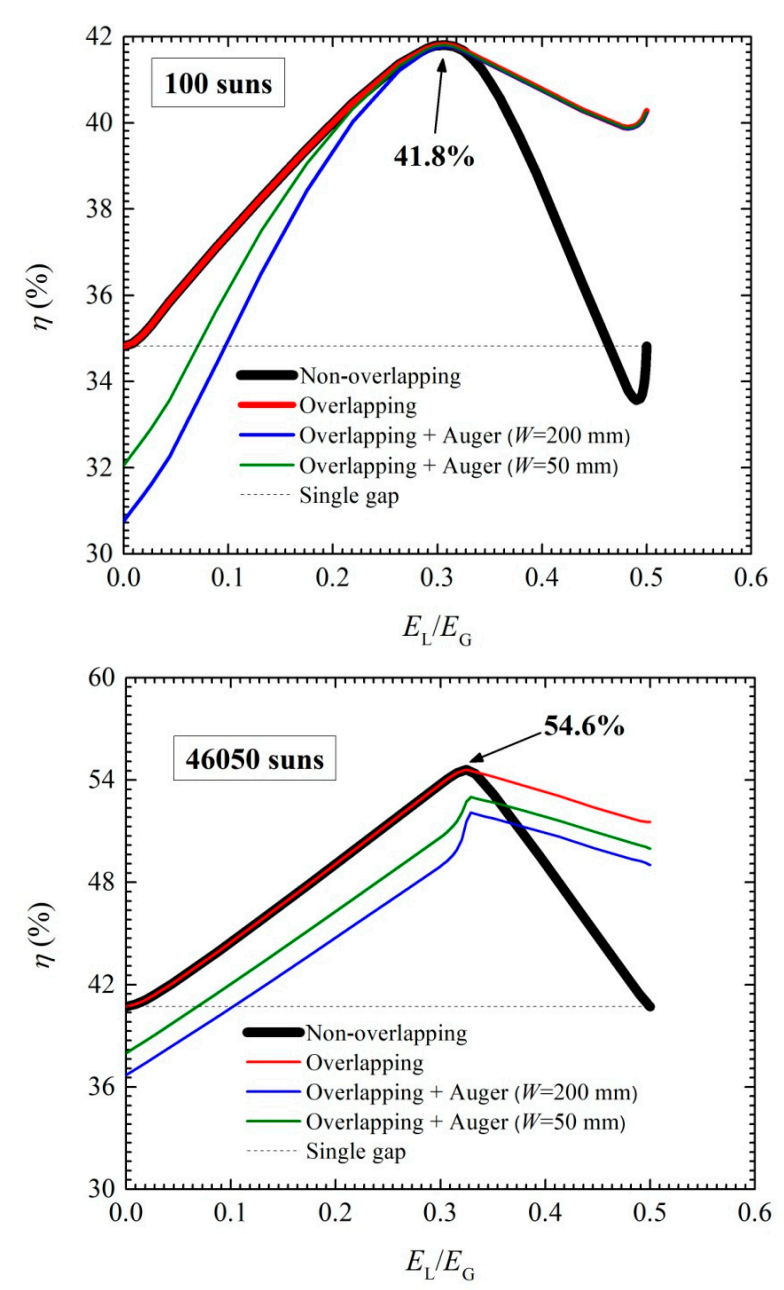

Figure 4. Limit efficiency of silicon IBSC at 100 (top) suns and 46,050 suns (bottom) for different IB positions with and without overlapping and with and without Auger recombination (discussed in Section 3.3). The value of $\gamma$ is optimized to maximize the efficiency at every $E_{L}$ and the limit efficiency of a conventional silicon solar cell is shown for comparison. 


\subsection{Auger Recombination}

The discussions in Sections 3.1 and 3.2 have been focused on the results obtained for the radiative limit. When Auger recombination is included non-radiative losses reduce the efficiency of silicon solar cells without IB (case in which $E_{L}=0$ ) by approximately four points for all the considered irradiances: 1100 and 46,050 suns. However, the reduction in efficiency due to Auger recombination is less severe in IBSCs. Indeed, at 1 and 100 suns, the efficiency in silicon IBSC remains intact for $E_{L}$ higher than the optimum value in which the maximum efficiency is reached. All these results are shown in Figures 3 and 4 and the most remarkable ones are summarized in Table 1.

Table 1. Summary of the limit efficiencies for a single gap silicon solar cell and a silicon IBSC including the effects of Auger recombination considering a cell thickness of $200 \mu \mathrm{m}$.

\begin{tabular}{ccccc}
\hline & Si Solar Cell & Si Solar Cell & Si-IBSC & Si-IBSC \\
\hline & Maximum efficiency & Auger losses & Maximum efficiency & Auger losses \\
\hline 1 sun & $30.4 \%$ & 4 points & $30.3 \%\left(\gamma=0 E_{\mathrm{L}} / E_{\mathrm{G}}=0.29\right)$ & - \\
100 suns & $34.8 \%$ & 4 points & $41.8 \%\left(\gamma=0 E_{\mathrm{L}} / E_{\mathrm{G}}=0.3\right)$ & - \\
suns & $40.7 \%$ & 4 points & $54.6 \%\left(\gamma=0 E_{\mathrm{L}} / E_{\mathrm{G}}=0.32\right)$ & 5 points $\left(E_{\mathrm{L}} / E_{\mathrm{G}}=0.3\right)$ \\
\hline
\end{tabular}

The reason why Auger recombination affects IBSCs less is because the presence of the IB in these cells already introduces an additional radiative recombination that, at one sun, is higher than the recombination introduced by Auger and, at 100 suns, is comparable. This is illustrated in Figure 5, where higher recombination rates are manifested as higher reductions in the output voltage, since this is the parameter more sensitive to that (the current density is not). At maximum concentration, the radiative recombination introduced by the IB is reduced enough to obtain no significant reduction in the output voltage (black triangles in Figure 5). So, the performance of IBSCs can be affected by the additional recombination introduced by Auger at that concentration (blue symbols in Figure 5).

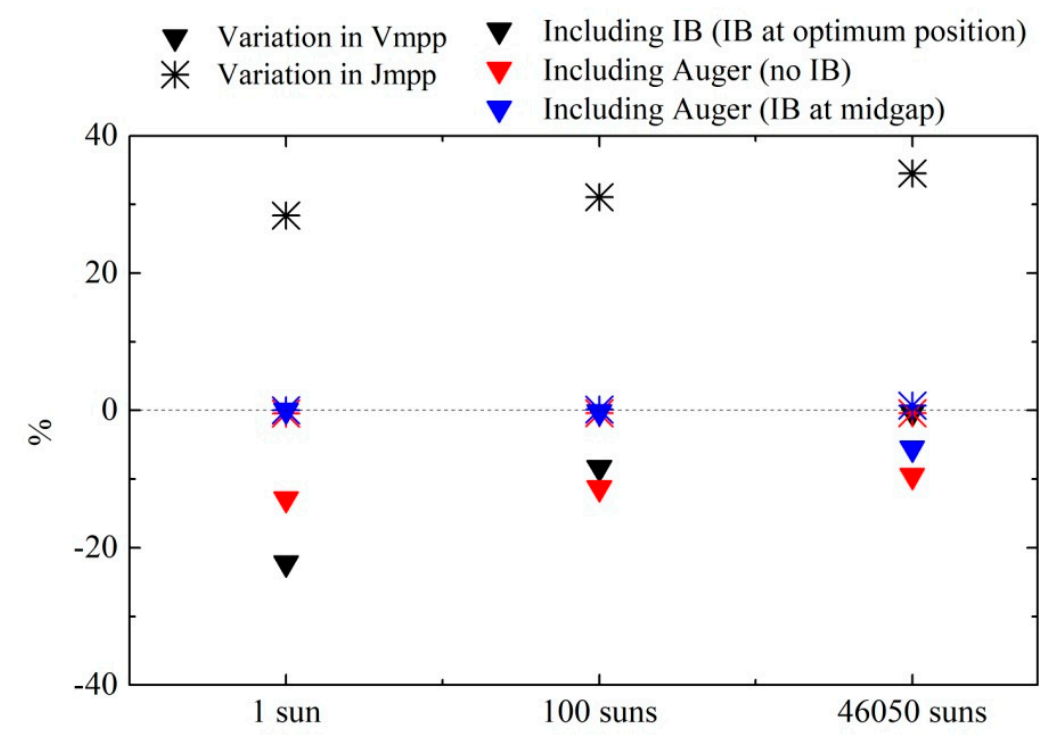

Figure 5. Relative variation of the voltage and current density at the maximum power point produced when an IB (black symbols) and Auger recombination are included. The effect of including Auger recombination is evaluated when there is no IB (red symbols) and when there is an IB at the middle of the bandgap (blue symbols).

Figure 5 is also useful to understand why the inclusion of an IB does not always provide a gain in efficiency, despite providing an increase in current density (black stars in Figure 5). This is the case of silicon IBSC at one sun, where the reduction in power due to the relative reduction in voltage is 
slightly higher than the increase in power due to the relative increase in current density. When the concentration increases from one sun to 46,050 suns, the efficiency increases mainly due to the increase in voltage, which approaches the voltage of a conventional cell without IB. The current density at the maximum power point also shows an increase with concentration, which is also related to the fact that under concentration higher FF are obtained (in the ideal cases with zero series resistances).

\section{Conclusions}

In this work the efficiency of silicon-based IBSCs under different concentrations of sunlight is studied, including for some realistic parameters such as overlapping in absorption coefficients and non-radiative Auger recombination. Results reveal that silicon non-ratchet IBSCs can surpass the limit efficiency of conventional silicon solar cells only under concentrated sunlight. The maximum efficiency of silicon IBSCs is achieved for an optimum bandgap distribution $\left(E_{H}\right.$ and $\left.E_{L}\right)$, assuming selective absorption coefficients. However, if non-optimum bandgaps are considered, overlapping absorption coefficients can lead to higher efficiencies than selective ones. The performance of silicon IBSC is less affected by Auger recombination than conventional silicon cells except if extremely high concentration is used to illuminate the devices.

Author Contributions: A.M. proposed to study the impact of non-overlapped absorption coefficients in IBSCs based on silicon and guided the work. E.L. did the calculations and wrote the paper. E.A. and A.L. revised the results and contributed to the discussions. All authors have read and agreed to the published version of the manuscript.

Funding: This research was funded by the Spanish Ministerio de Ciencia, Innovación y Universidades through the Project 2GAPS (TEC2017-92301-EXP); the Comunidad de Madrid through the Project MADRID-PV2-CM (S2018/EMT-4308), supported with FEDER funds; and the European Commission through the NGCPV Project (Grant Number 283798). Esther López acknowledges an Atracción del Talento Fellowship (2019-T2/AMB-12959) funded by the Comunidad de Madrid. Elisa Antolín acknowledges a Ramón y Cajal Fellowship (RYC-2015-18539) funded by the Spanish Science Ministry.

Disclaimer: The results presented in this paper in Sections 3.1 and 3.2 were presented at the 2014 IEEE 40th Photovoltaics Specialist Conference under the title "Limiting Efficiency of silicon intermediate band solar cells". However, due to technical problems beyond the responsibility of the authors, they are not accessible and have included for completitude. The study about the impact of overlapping absorption coefficients in intermediate band solar cells working in the radiative limit is notably expanded by Krishna and Krich in "Increasing efficiency in intermediate band solar cells with overlapping absorptions" published in 2016 in the Journal of Optics, Volume 18 , where many other materials than silicon are studied.

Conflicts of Interest: The authors declare no conflict of interest.

\section{References}

1. Luque, A.; Martí, A. Increasing the Efficiency of Ideal Solar Cells by Photon Induced Transitions at Intermediate Levels. Phys. Rev. Lett. 1997, 78, 5014-5017. [CrossRef]

2. Luque, A.; Martí, A. The Intermediate Band Solar Cell: Progress toward the Realization of an Attractive Concept. Adv. Mater. 2010, 22, 160-174. [CrossRef] [PubMed]

3. Yoshida, M.; Ekins-Daukes, N.; Farrell, D.J.; Phillips, C.C. Photon ratchet intermediate band solar cells. Appl. Phys. Lett. 2012, 100, 263902. [CrossRef]

4. Mott, N.F. Metal-Insulator Transition. Rev. Mod. Phys. 1968, 40, 677-683. [CrossRef]

5. Luque, A.; Martí, A.; Antolin, E.; Tablero, C. Intermediate bands versus levels in non-radiative recombination. Phys. B Condens. Matter 2006, 382, 320-327. [CrossRef]

6. Tablero, C.; Crespo, C.T. Effects of the impurity-impurity and impurity-host interactions on the charge density and the related processes. Phys. B Condens. Matter 2009, 404, 4023-4028. [CrossRef]

7. Crespo, C.T. Effects of the impurity-host interactions on the nonradiative processes in ZnS:Cr. J. Appl. Phys. 2010, 108, 93114. [CrossRef]

8. Krich, J.J.; Halperin, B.I.; Aspuru-Guzik, A. Nonradiative lifetimes in intermediate band photovoltaics-Absence of lifetime recovery. J. Appl. Phys. 2012, 112, 13707. [CrossRef] 
9. Olea, J.; Pastor, D.P.; Toledano-Luque, M.; Andres, E.S.; Mártil, I.; González-Díaz, G. High Quality Ti-Implanted Si Layers Above Solid Solubility Limit. In Proceedings of the 2009 Spanish Conference on Electron Devices, Santiago de Compostela, Spain, 11-13 February 2009; pp. 38-41.

10. Olea, J.; Pastor, D.P.; Del Prado, Á; García-Hemme, E.; García-Hernansanz, R.; Mártil, I.; González-Díaz, G. Ruling out the impact of defects on the below band gap photoconductivity of Ti supersaturated Si. J. Appl. Phys. 2013, 114, 53110. [CrossRef]

11. García-Hemme, E.; García-Hernansanz, R.; Olea, J.; Pastor, D.P.; Del Prado, Á.; Mártil, I.; González-Díaz, G. Sub-bandgap spectral photo-response analysis of Ti supersaturated Si. Appl. Phys. Lett. 2012, 101, 192101. [CrossRef]

12. Zhou, Y.; Liu, F.; Song, X. The insulator-to-metal transition of Co hyperdoped crystalline silicon. J. Appl. Phys. 2013, 113, 103702. [CrossRef]

13. Ertekin, E.; Winkler, M.T.; Recht, D.; Said, A.J.; Aziz, M.J.; Buonassisi, T.; Grossman, J.C. Insulator-to-Metal Transition in Selenium-Hyperdoped Silicon: Observation and Origin. Phys. Rev. Lett. 2012, 108, 026401. [CrossRef] [PubMed]

14. Said, A.J.; Recht, D.; Sullivan, J.T.; Warrender, J.; Buonassisi, T.; Persans, P.D.; Aziz, M.J. Extended infrared photoresponse and gain in chalcogen-supersaturated silicon photodiodes. Appl. Phys. Lett. 2011, 99, 73503. [CrossRef]

15. Sullivan, J.T.; Simmons, C.B.; Krich, J.J.; Akey, A.J.; Recht, D.; Aziz, M.J.; Buonassisi, T. Methodology for vetting heavily doped semiconductors for intermediate band photovoltaics: A case study in sulfur-hyperdoped silicon. J. Appl. Phys. 2013, 114, 103701. [CrossRef]

16. Persans, P.D.; Berry, N.E.; Recht, D.; Hutchinson, D.; Peterson, H.; Clark, J.; Charnvanichborikarn, S.; Williams, J.; DiFranzo, A.; Aziz, M.J.; et al. Photocarrier lifetime and transport in silicon supersaturated with sulfur. Appl. Phys. Lett. 2012, 101, 111105. [CrossRef]

17. Winkler, M.T.; Recht, D.; Sher, M.-J.; Said, A.J.; Mazur, E.; Aziz, M.J. Insulator-to-Metal Transition in Sulfur-Doped Silicon. Phys. Rev. Lett. 2011, 106, 178701. [CrossRef] [PubMed]

18. Antolín, E.; Martí, A.; Olea, J.; Pastor, D.P.; González-Díaz, G.; Mártil, I.; Luque, A. Lifetime recovery in ultrahighly titanium-doped silicon for the implementation of an intermediate band material. Appl. Phys. Lett. 2009, 94, 42115. [CrossRef]

19. González-Díaz, G.; Olea, J.; Mártil, I.; Pastor, D.P.; Martí, A.; Antolín, E.; Luque, A. Intermediate band mobility in heavily titanium-doped silicon layers. Sol. Energy Mater. Sol. Cells 2009, 93, 1668-1673. [CrossRef]

20. Krishna, A.; Krich, J.J. Increasing efficiency in intermediate band solar cells with overlapping absorptions. J. Opt. 2016, 18, 74010. [CrossRef]

21. Cuadra, L.; Martí, A.; Luque, A. Influence of the Overlap between the Absorption Coefficients on the Efficiency of the Intermediate Band Solar Cell. IEEE Trans. Electron Devices 2004, 51, 1002-1007. [CrossRef]

22. Yablonovitch, E.; Cody, G. Intensity enhancement in textured optical sheets for solar cells. IEEE Trans. Electron Devices 1982, 29, 300-305. [CrossRef]

23. Campbell, P.; Green, M.A. The limiting efficiency of silicon solar cells under concentrated sunlight. IEEE Trans. Electron Devices 1986, 33, 234-239. [CrossRef]

24. Martí, A.; Araújo, G.L. Limiting efficiencies for photovoltaic energy conversion in multigap systems. Sol. Energy Mater. Sol. Cells 1996, 43, 203-222. [CrossRef]

25. Araujo, G.; Martí, A. Limiting efficiencies of GaAs solar cells. IEEE Trans. Electron Devices 1990, 37, $1402-1405$. [CrossRef]

26. Pusch, A.; Ekins-Daukes, N.J. Voltage Matching, Étendue, and Ratchet Steps in Advanced-Concept Solar Cells. Phys. Rev. Appl. 2019, 12, 044055. [CrossRef]

(C) 2020 by the authors. Licensee MDPI, Basel, Switzerland. This article is an open access article distributed under the terms and conditions of the Creative Commons Attribution (CC BY) license (http://creativecommons.org/licenses/by/4.0/). 